\title{
Úlcera corneal complicada secundaria a rosácea ocular
}

\author{
Dr. Diego Fernando Carrión Jaramillo ${ }^{1}$ \\ Dr. Miguel Andrés Silva Muñoz ${ }^{2}$ \\ Dr. Carlos René León Roldán
}

\section{Resumen}

La rosácea es una enfermedad de etiología aún poco comprendida, presenta respuesta inflamatoria del sistema inmune y de las vías neurovasculares, a ciertos desencadenantes que conllevan a un proceso inflamatorio crónico. Más del 50 \% de los pacientes con rosácea presentan manifestaciones oculares, que de no tratarse adecuadamente, evolucionan con un sinnúmero de complicaciones que afectan la calidad de vida. Se presenta el caso de una paciente de treinta y un años con diagnóstico de rosácea exacerbada, presentada ulceración corneal en ojo izquierdo (OI) y perforación, requiriendo trasplante corneal. Realizamos una revisión bibliográfica haciendo énfasis en las diferentes afecciones oculares y su tratamiento con el propósito de evitar la aparición de complicaciones severas.

Palabras clave: rosácea, úlcera, queratitis, descemetocele, perforación, queratoplastia.

\section{Abstract}

Rosacea is a disease of not very well understood etiology, but with a great inflammatory response of the immune system and neurovascular pathways to certain triggers that lead to a chronic inflammatory process. More than $50 \%$ of patients with rosacea have ocular manifestations, which if not adequately treated, evolve with a number of complications that affect the quality of life. We present the case of a thirty one year old patient with a diagnosis of exacerbated rosacea presented with corneal ulceration in the left eye and perforation requiring a corneal transplant. We conducted a literature review emphasizing the different ocular conditions and their treatment in order to avoid the appearance of severe complications.

Keywords: rosacea, ulcer, keratitis, descemetocele, perforation, keratoplasty

\footnotetext{
${ }^{1}$ Médico y cirujano residente de tercer año de la Escuela Superior de Oftalmología - Centro Oftalmológico León.

${ }^{2}$ Médico y cirujano residente de tercer año de la Escuela Superior de Oftalmología - Centro Oftalmológico León.

${ }^{3}$ Médico y cirujano oftalmólogo. Director de la Escuela Superior de Oftalmología - Centro Oftalmológico León
} 


\section{Introducción}

La rosácea es un trastorno inflamatorio acneiforme crónico que se caracteriza por eritema facial central transitorio o persistente, vasos sanguíneos telangiectásicos y, a menudo, pápulas y pústulas. Aunque la etiología no se comprende completamente, se cree se debe a una respuesta inflamatoria aumentada del sistema inmune y de las vías neurovasculares a ciertos desencadenantes que conllevan a un proceso inflamatorio crónico (1). Se ha clasificado la rosácea en cuatro subtipos por el National Rosacea Society Expert: eritematotelangiectásica, papulopustular, fimatosa y ocular. Más del 50 \% de los pacientes presentan manifestaciones oculares, estas incluyen desde una irritación leve, sensación de cuerpo extraño, sequedad, visión borrosa hasta queratitis. Generalmente, se acompaña de blefaroconjuntivitis. Otros hallazgos consisten en telangiectasias en el borde palpebral y conjuntiva, tilosis, orzuelos y chalazión, infiltrados corneales, úlceras corneales, cicatrices corneales y vascularización, que si no se tratan adecuadamente pueden evolucionar a perforación corneal (2).
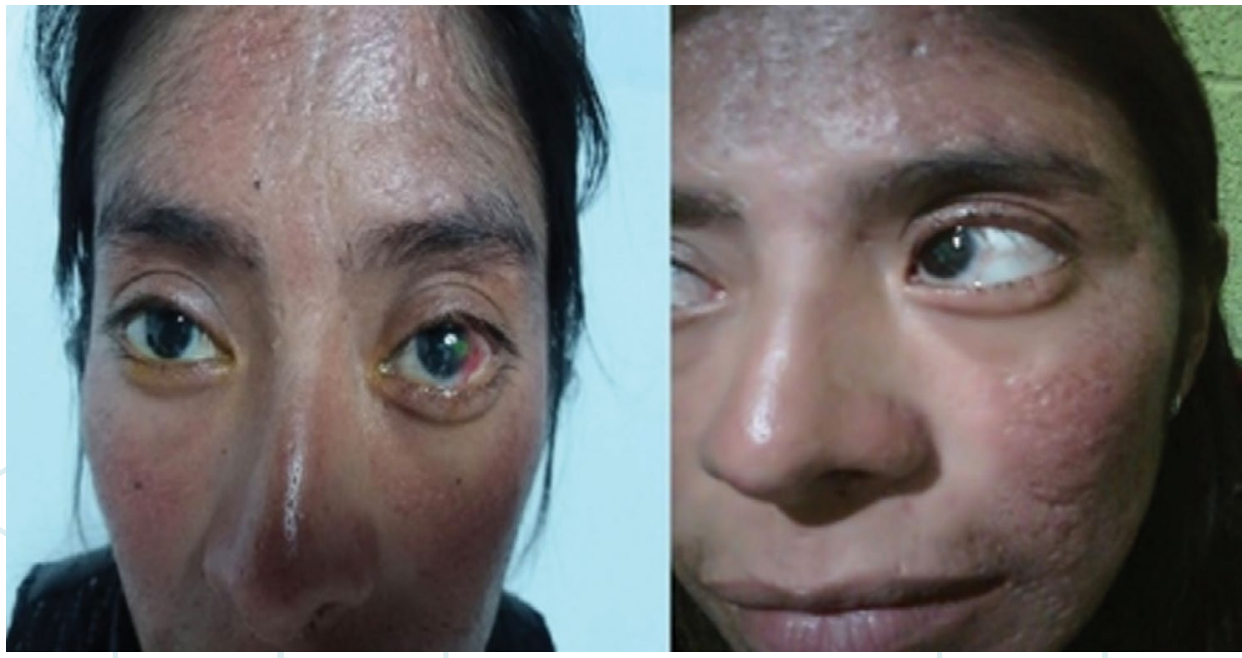

Imagen 1. A. Rosácea dérmica y ocular activa; B. Rosácea dérmica y ocular posterior a tratamiento.
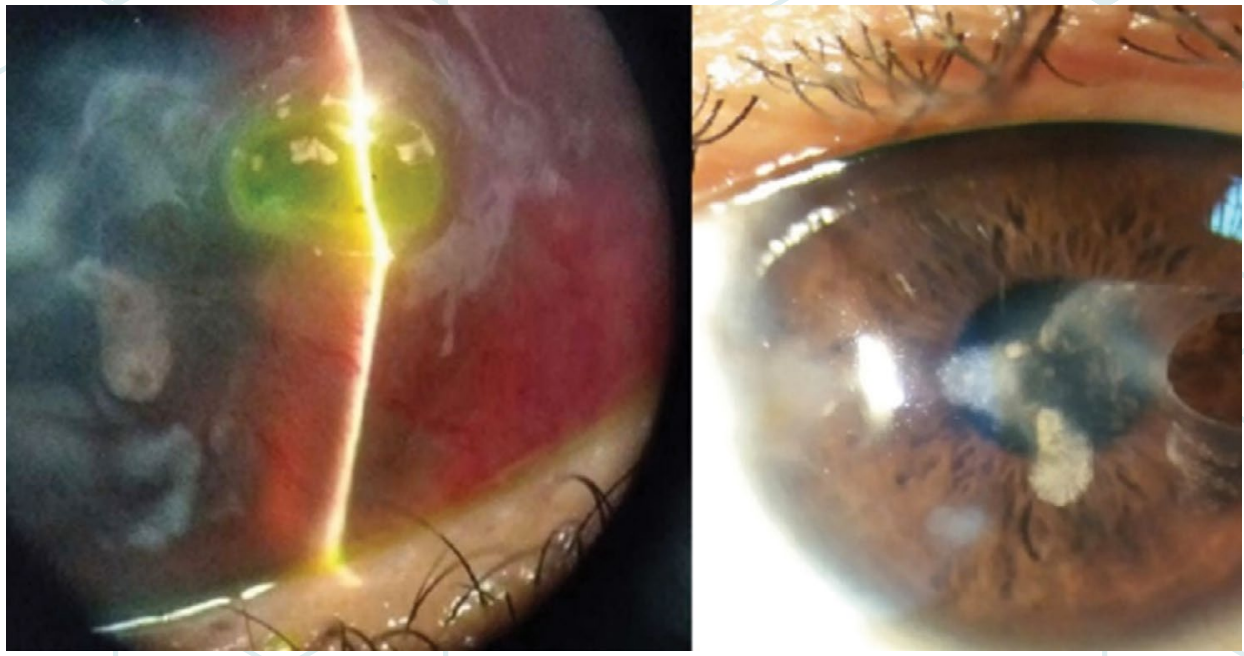

Imagen 2. A. Úlcera corneal temporal de ojo izquierdo con importante adelgazamiento; B. Descemetocele de $3 \times 4 \mathrm{~mm}$ y leucoma central y periférico secundario a úlcera corneal. 
Reporte de caso

Paciente femenina de treinta y un años con diagnóstico de rosácea dérmica y ocular de tres años de evolución sin tratamiento actual. Acude a consulta por disminución de agudeza visual de ojo izquierdo (OI), ojo rojo, dolor ocular, secreción blanquecina, de quince días de evolución. Al examen médico se observa eritema centro facial con engrosamiento de piel acompañado de pápulas, pústulas y telangiectasias de predominio nasal.

\section{Al examen oftalmológico} presenta agudeza visual (AV) ojo derecho (OD): 20/100 que corrige a 20/20, y Ol: $20 / 400$ que no corrige. Al evaluar anexos oculares se aprecia tilosis, collaretes y disfunción de las glándulas de Meibomio (DGM). Biomicroscopia: en OD múltiples leucomas con áreas de adelgazamiento que no tiñen con fluoresceína, sin compromiso del eje visual; $\mathrm{OI}$ con hiperemia conjuntival en cuadrante temporal, secreción amarillenta, leucoma central, úlcera corneal temporal de $3 \times 4 \mathrm{~mm}$ con adelgazamiento del 90 \% que tiñe con fluoresceína; resto de examen oftalmológico sin hallazgos relevantes.

Se inicia manejo para úlcera corneal en OI con ciprofloxacina $0.3 \%$, dexametasona 0.1 $\%$, hialuronato de sodio 0.5 $\%$, ácido poliacrílico $2 \%, y$ vendaje con lente de contacto blando. Además, doxiciclina, metronidazol ungüento $0.75 \%$, y fototerapia con luz pulsada intensa. Como secuela se desencadena un descemetocele temporal, que finalmente progresa a perforación corneal espontánea sin causa aparente con prolapso de iris que produce efecto de taponamiento.

Debido al tamaño de la perforación se realizó queratoplastia tectónica y recubrimiento conjuntival en $\mathrm{OI}$

\section{Discusión}

La rosácea es una enfermedad inflamatoria crónica con importante compromiso oftalmológico. Los síntomas y signos son muy variados, siendo el disconfort ocular el motivo principal de consulta, razón por la cual el oftalmólogo juega un papel importante para el diagnóstico y tratamiento oportuno. La rosácea puede producir consecuencias menores, así como progresar agresivamente hasta provocar perforación corneal (3). La queratitis ulcerativa periférica por lo general es de difícil manejo y resolución. El presente caso resalta la importancia del oftalmólogo en su capacidad de realizar un examen físico completo, diagnóstico oportuno y tratamiento médico, no solo del componente ocular sino además del compromiso cutáneo (4). Está descrito que la severidad de la rosácea no tiene relación directa con la severidad del compromiso ocular, sin embargo, en el presente caso se demuestra cómo el tratamiento integral influyó positivamente en el control de los síntomas (5). Se identificó el difícil acceso al servicio de salud, la pobre educación de la paciente en relación a su patología de base, factores ambientales, ojo seco, blefaroconjuntivitis, disfunción de las glándulas de Meibomio, cronicidad de los síntomas, diagnóstico tardío y la falta de oportunidad para el tratamiento como factores desencadenantes que conllevaron a un adelgazamiento y perforación corneal. La razón de presentar este caso es la afección visual tan severa de la paciente, ya que de haber sido detectada y tratada oportunamente, se hubiera podido evitar la discapacidad visual y el compromiso de su calidad de vida $(6,7)$. 


\section{Conclusión}

La prevalencia exacta de la afección ocular de los pacientes con rosácea, ha sido difícil de determinar. Existen registros bibliográficos que la reportan con una variación tan grande como del $3 \%$ al $58 \%$. La enfermedad puede iniciar con afección solo ocular y mantenerse así por muchos años, razón por la cual el tratamiento es inespecífico. Las alteraciones oculares son independientes de la rosácea cutánea, sin embargo, hay una fuerte relación entre las manifestaciones oculares y el eritema facial. La queratitis por rosácea tiene un pronóstico desfavorable y en casos severos produce ceguera por la opacidad corneal.

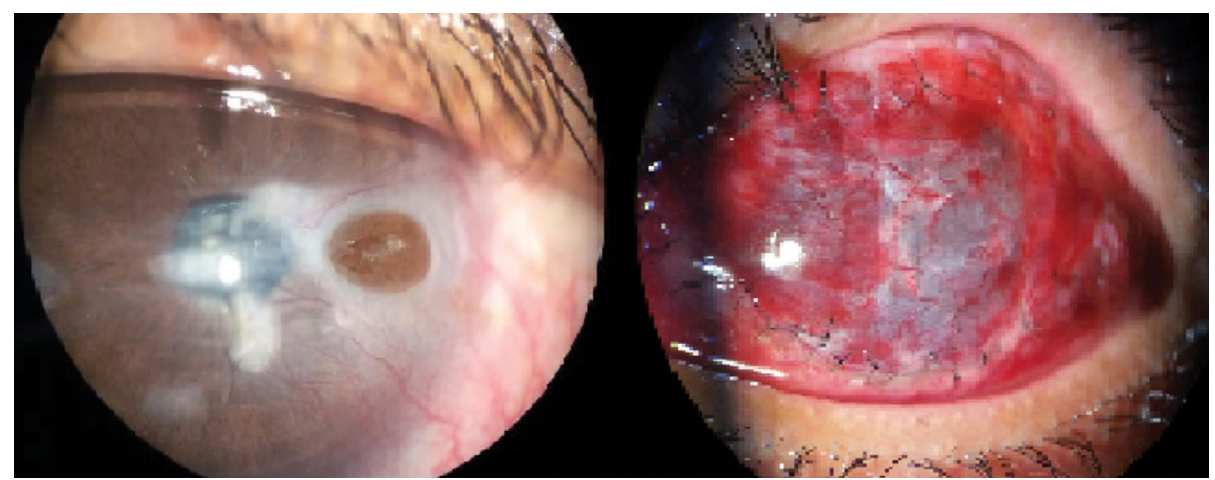

Imagen 3. A. Perforación corneal con prolapso de iris de ojo izquierdo; B. Queratoplastia Corneal más recubrimiento conjuntival ojo izquierdo

\section{Referencias}

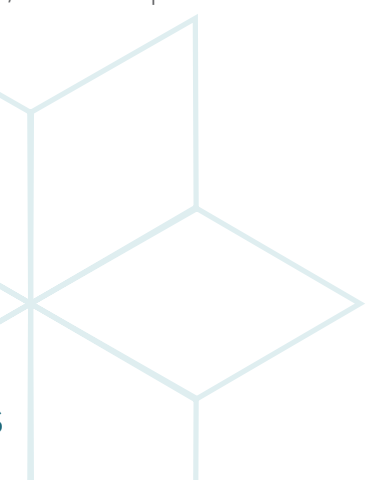

1. Wilkin J, Dahl M, Dermar M et al. Standard classification of rosacea: report of the National Rosacea Society Expert Committee on the Classification and Staging of Rosacea. J Am A Dermatol. 2002;46:586-587.

2. Diamantis S, Waldorf HA. Rosacea: Clinical presentation and pathophysiology. J Drugs Dermatol. 2006;5(1):8-12.

3. Aloi F, Tomasini C, Soro E, Pippione M. The clinicopathologic spectrum of rhinophyma. J Am Acad Dermatol. 2000;42(3)468-472.

4. Crawford GH, Pelle MT, James WD. Rosacea: 1. Ethiology, pathogenesis, and subtype classification. J Am A Dermatol. 2004;51:327-341.

5. Bamford JT, Gessert CE, Renier CM. Measurement of the severity of rosacea. J Am Acad Dermatol. 2004;51(5):697-703.

6. Higgins E, du Vivier A. Alcohol intake and other skin disorders. Clin Dermatol. 1999;17(4):437-441.

7. Neumann E, Frithz A. Capillaropathy and capillaroneogenesis in the pathogenesis of rosacea. Int J Dermatol. 1998;37(4):263-266. 